\title{
A Novel Approach for Localization of Moving Target Nodes in Wireless Sensor Networks
}

\author{
Parulpreet Singh ${ }^{1}$, Arun Khosla ${ }^{2}$, Anil Kumar ${ }^{3}$ and Mamta Khosla ${ }^{4}$ \\ ${ }^{1,2,4}$ Department of Electronics and Communication Engineering, National \\ Institute of Technology, Jalandhar, India \\ ${ }^{3}$ Department of Electronics and Communication Engineering, Chandigarh \\ College of Engineering and Technology, Chandigarh, U.T., India \\ parulpreet89@gmail.com
}

\begin{abstract}
Localization of a target node is a fundamental and important requirement in wireless sensor network (WSN) because the sensor data is useless if we don't know the exact location of the occurring event. So, most of the applications in WSNs requires the geographical location of the sensor nodes. This paper proposes the application of PSO based computation intelligence algorithm for distributed optimal localization of randomly moving target nodes. Anchor nodes are deployed at the edges of the sensing field. The performance based results on experimental mobile sensor network data demonstrate the effectiveness of the proposed algorithms by comparing the performance in terms of the number of nodes localized, localization accuracy and scalability.
\end{abstract}

Keywords: Particle Swarm Optimization, Localization, Wireless Sensor Networks

\section{Introduction}

WSNs, the deployment of sensor nodes in the real world environment is done for determining some physical behavior. These sensor nodes are very small devices having low cost and computational capabilities. Environmental monitoring, physical phenomenon like sound, temperature and light monitoring, habitat monitoring, underwater acoustic monitoring are some of the applications of sensor networks [1,2]. Apart from this, WSNs have many research challenges like hardware and OS, deployment, localization, QOS, network security and many more. Localization of nodes in WSNs is very important because the sensor data is useless if we don't know the exact location of the occurring event. The simplest method for getting location of a node is to equip every node with a Global Positioning System (GPS), but it become expensive in case of network having large number of nodes [3]. Many algorithms have been proposed in the literature for cost effective and efficient localization but most of the algorithms are application specific and not suitable for wide range of Wireless Sensor Networks. Some basic localization techniques can be used to find the location of the sensor nodes which can be classified as range-based and rangefree localization techniques. Some of the Range-Based localization techniques are Received Signal Strength Indicator (RSSI), Angle of Arrival (AoA), Time of Arrival (ToA) and Time Difference of Arrival (TDoA) [4, 5]. Similarly, Multidimensional Scaling (MDS), AdHoc Positioning System (APS) and Distance Vector Hop (DV-Hop) are some of the range free localization techniques $[6,7]$. The nodes whose location is being determined by using prior knowledge of other nodes is considered as target nodes and the other nodes whose locations are known to us is considered as anchor nodes. Anchor node beacon messages can be used to estimate the position of target nodes but it requires more energy in terms of power and have high communication cost. If wrong location is estimated by an algorithm, then this

Received (July 5, 2017), Review Result (September 29, 2017), Accepted (October 3, 2017) 
error will be propagated throughout the network. So, localization accuracy should be high. To achieve high accuracy, some of the bio inspired based location optimization algorithms are available in the literature.

In this paper, localization is done using single anchor node only and the target nodes are moving randomly in the network area. Once moving target node falls under the range of one anchor node, the distance is calculated between anchor and target node using RSS measures. Then two virtual anchor nodes are projected inside the network at the distance which is equal to the anchor and in-range target node. Centroid is calculated using anchor and two virtual anchor nodes and PSO based bio inspired technique is used to optimize the estimated location.

The organization of the rest of paper is as follows: Literature review on localization is given in Section 2. Section 3 gives the idea about PSO based bio inspired algorithm and methodology used in this paper for localization. Section 4 gives the simulation results and discussion and Section 5 gives the future scope of the work.

\section{Related Work}

Generally, there are a number of anchor nodes in the sensor network and the location of each anchor node is known to us. Due to cost and complexity constrains, some of the node locations are not known to us. These nodes location is estimated by using location of anchor nodes. Many localization approaches were given by researchers in the past years [8, 17-23]. In this paper our major focus is on localization methods using distance measurements and estimated location optimization using PSO based bio inspired algorithm in mobility based scenario.

\section{A. Measurement of Distance}

Measurement of distance between anchor and target nodes may be obtained by received signal strength (RSS) information. Another ways of getting distance between anchor and target is ToA, TDoA and Round Trip Time of Flight (RToF). In the above mentioned techniques, some of the techniques have very accurate synchronization between anchor and target. But due to very high propagation speed, less error in the measurement of propagation time leads to large error in the measurement of the distance.

\section{B. Estimation of Coordinates in Mobility Based Scenario.}

In literature many coordinates' estimation techniques for static and dynamic target nodes are available. The main use of these techniques is to find out physical coordinates of the target nodes in the network area. For situations in which it is not practical or cost efficient to use GPS or anchor nodes, An Anchor-Free Mobile Geographic Distributed Localization (MGDL) [9] algorithm for wireless sensor networks has been proposed which obtains better coverage than Anchor Based mobile localization [9]. The MGDL has flexible communication overhead for both high-mobility and low-mobility nodes. Another Method MACL (mobile anchor Centroid localization) [10], a frequency based approach, which uses a single mobile anchor node to move in the sensing field and broadcast its current position periodically. The simulation experiments reveal that the MACL technique can provide accurate localization even when memory limits are severe, the seed density is low, and network transmissions are highly irregular. Garg, V. and Jhamb, M. [11] proposed RSS based approach to locate the position of the mobile node (node moving in $2 \mathrm{D}$ environment and are not aware of its position in the network). They positioned anchor nodes at the vertices and the target node is moving freely inside the square area. The goal of the work is to track the location of mobile node by applying Heron's Formula on the triangles formed inside the square. They conclude that the communication overhead is less while computation overhead is more in localization process of the mobile target node. 
To enhance the precision and reduce the complexity, applications of CI based algorithms have been proposed as location optimization techniques. For static scenarios, Genetic Algorithms (GA) and other Stochastic Algorithm (SA) based localization algorithms are available in the literature. Raghavendra V. Kulkarni [12] presented PSO with its application in WSNs Localization. A. Gopakumr and Lillykutty Jacob [13] proposed a global optimization strategy using swarm intelligence and the node localization is done using this strategy. Mean square range error is taken as objective function in this technique. The authors suggested that PSO algorithm is exceptionally reasonable for resource constrained environment due to its simple execution. Kumar A. et al. [14] proposed the application of H-Best PSO (Particle Swarm Optimization) and BBO (Biogeography Based Optimization) for enhancement of evaluated location information of arbitrarily deployed target nodes. The HPSO calculation was demonstrated for mature and quick convergence and $\mathrm{BBO}$ was utilized for more precision. Kumar A. et al. [15] proposed 3D node localization strategies for anisotropic WSNs by utilizing the applications of HPSO and BBO, termed as range-free HPSO (RFHPSO) and range-free BBO (RFBBO). In the proposed technique, additional hardware is not required for calculating distance amongst target and anchor nodes. Edge weights amongst anchor and target nodes are considered for estimating the location of target node. Likewise, the edge weights are modeled by utilizing Fuzzy Logic System (FLS) to limit the computational intricacy and these weights are further enhanced.

\section{Node Localization using PSO}

An evolutionary computation technique named particle swarm optimization was developed by Kennedy and Eberhart [16]. This technique is based on the behavior of flocking birds. PSO is a computationally efficient algorithm and also it is easy to implement. The solutions named particles are employed in the search space with random locations. The objective function is calculated corresponding to the particles random locations. Every particle move towards the best position to gather its pBest (particle best) and gBest (globally best) position by obeying some rules which are motivated by flocking birds and fish schools $[16,14]$. The methodology followed for localization using PSO is given in Figure 1.

$$
\mathrm{d}_{\mathrm{i}}=\sqrt{\left(\mathrm{x}-\mathrm{x}_{\mathrm{i}}\right)^{2}-\left(\mathrm{y}-\mathrm{y}_{\mathrm{i}}\right)^{2}} \ldots \ldots
$$

Each localizable node can calculate its distance from the neighboring anchor node by above equation (1)

$$
\widehat{\mathrm{d}_{1}}=\mathrm{d}_{\mathrm{i}}+\mathrm{n}_{\mathrm{i}} \ldots \ldots \ldots \ldots \ldots \ldots \ldots \ldots \ldots \ldots
$$

Due to effect of noise the distance can be calculated by equation (2). Where $\mathrm{n}_{\mathrm{i}}$ is a Gaussian Noise.

$$
f(x, y)=\frac{1}{M} \sum\left(\sqrt{\left(x-x_{i}\right)^{2}-\left(y-y_{i}\right)^{2}}-\widehat{d_{1}}\right) .
$$

The equation $[14,15]$ of objective function is given by (3).

The basic flow of the localization process using PSO is shown in the Figure-1. In this paper, this technique is used for mobility based scenario. The target nodes are mobile and localization is done using single anchor node only. Figure- 2 shows the projection of virtual anchor nodes inside the sensing field. When a target comes under the range of single anchor node, two virtual anchor nodes are projected inside the sensing field at a distance equal to anchor and in-range target node.

Figure-3 shows the complete flow of localization process used in this paper. Initially, target nodes are randomly deployed in the sensing field and random mobility is given to these target nodes. The anchor nodes are deployed at the edges of the sensing field. Once a moving target node comes under the range of single anchor node, distance is calculated between anchor and in-range target node and two virtual anchor nodes are projected inside the sensing field, shown in Figure-2. Further, Centroid is calculated using anchor and virtual 
anchor nodes. Then optimization of estimated coordinated is done using PSO based bioinspired algorithm.

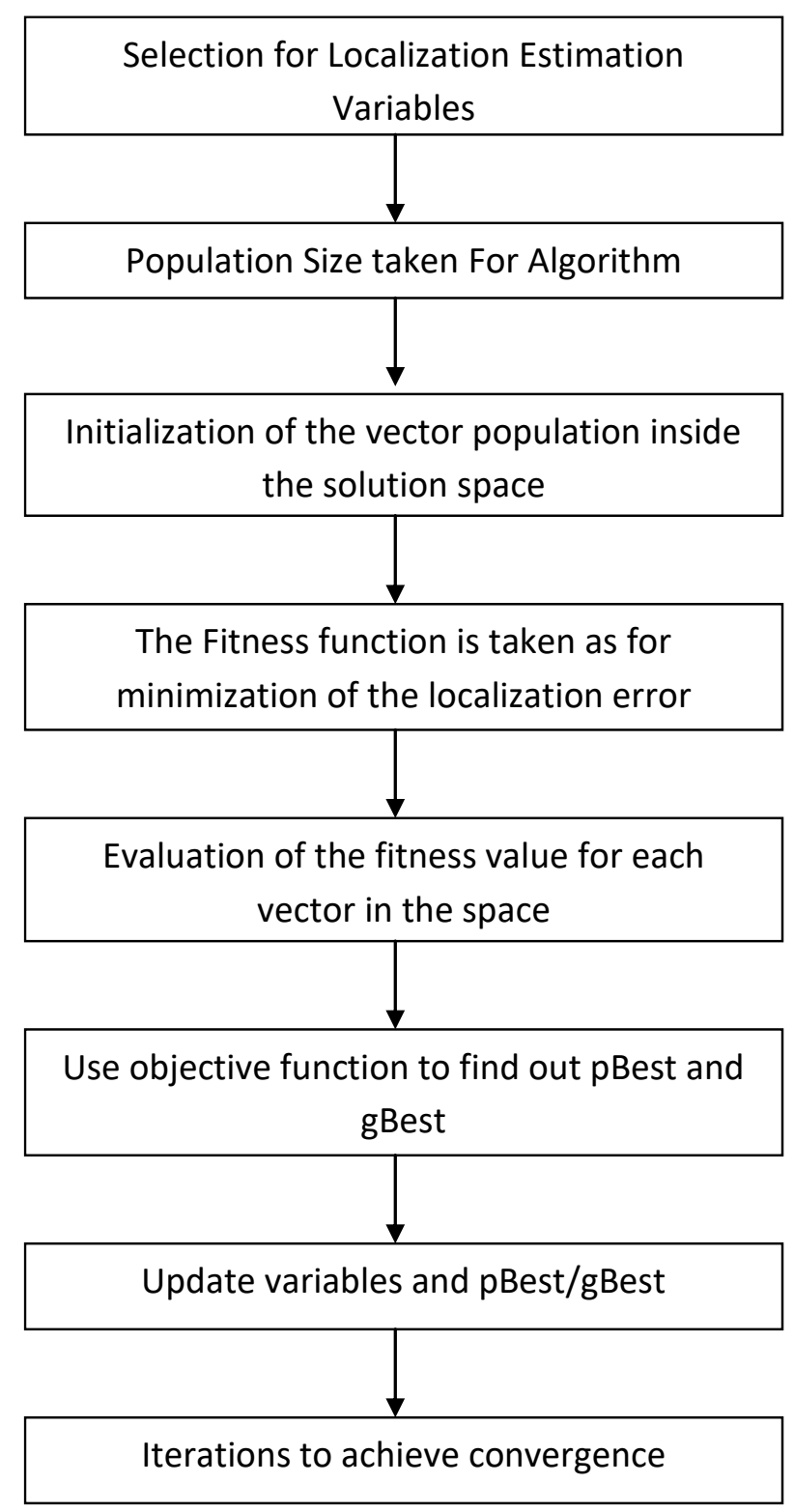

Figure 1. Localization Process using PSO

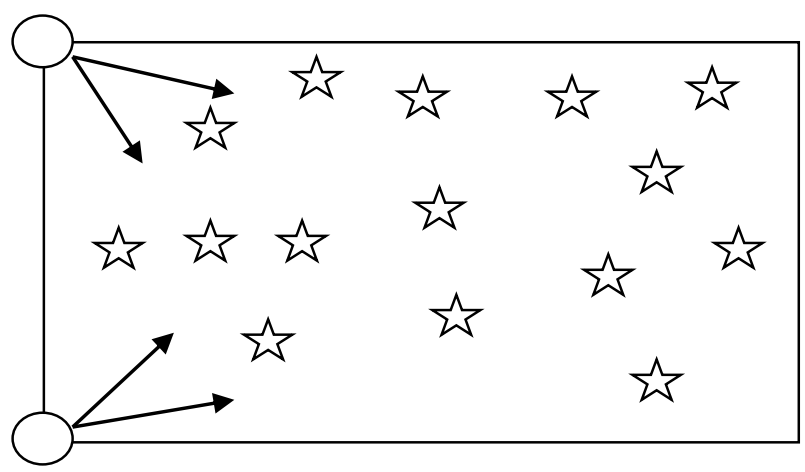

Figure 2. Projection of Virtual Anchor Nodes 


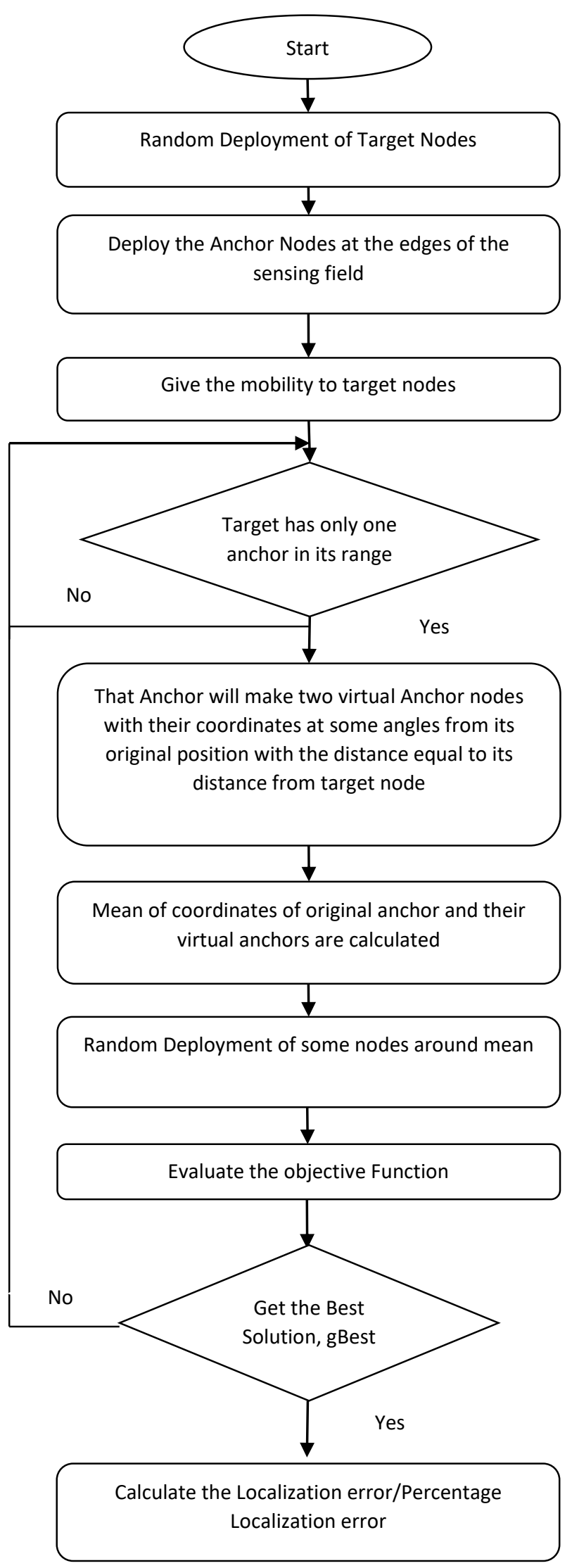

Figure 3. Methodology Followed for Localization Process using PSO 


\section{Simulation Results and Discussions}

In order to evaluate the performance of the proposed PSO based algorithm on mobility based scenarios, many simulations were performed on MATLAB software. These simulations were performed on PC having Intel core i3 processor and 4GB of RAM. The simulations have been done on small network of size $15 \times 151$ units. 10 mobile target nodes are deployed in the sensing field and 6 anchor nodes are deployed at the edges of the sensing field. The Other parameters used for simulation is shown in the Table 1.

Table 1. Simulation Parameters used for Localization using PSO

\begin{tabular}{|l|l|}
\hline Area & $15 X 15$ Units \\
\hline Anchor Nodes & 6 \\
\hline Target Nodes & 10 \\
\hline Mobility & Target/Random \\
\hline Range & 7 Units \\
\hline PSO Radius & 1 Unit \\
\hline Virtual Anchors & 2 per Anchor \\
\hline
\end{tabular}

The range in this paper is taken 7 units because by using range as 7 units, whole sensing field can be covered. Table 2 shows each target node coordinates at first positions.

Table 2. Target Node Coordinates at First Movement

\begin{tabular}{|l|l|l|}
\hline Node No. & \multicolumn{2}{|l|}{$\begin{array}{l}\text { Target Coordinates for First } \\
\text { Movement }\end{array}$} \\
\hline & X & Y \\
\hline 1 & 7.1572 & 4.6053 \\
\hline 2 & 14.6384 & 6.0746 \\
\hline 3 & 14.9896 & 6.7677 \\
\hline 4 & 1.3006 & 5.0534 \\
\hline 5 & 1.6413 & 0.2847 \\
\hline 6 & 1.0154 & 1.5203 \\
\hline 7 & 8.6956 & 8.9502 \\
\hline 8 & 14.3901 & 14.0977 \\
\hline 9 & 14.1993 & 12.8078 \\
\hline 10 & 5.0189 & 11.6376 \\
\hline
\end{tabular}

Table 3. Efficiently Localized Node for First Movement

\begin{tabular}{|l|l|l|l|l|}
\hline \multicolumn{5}{|l|}{ Efficiently Localize Node for First count } \\
\hline Localize Node Coordinates & Error & \% Error & Node Number \\
\hline 7.8056 & 4.5533 & 0.6505 & 4.3366 & 1 \\
\hline 1.6378 & 0.2822 & 0.0043 & 0.0286 & 5 \\
\hline 0.3978 & 1.7331 & 0.6532 & 4.3546 & 6 \\
\hline 14.5041 & 14.2740 & 0.2099 & 1.3993 & 8 \\
\hline 14.5380 & 12.6446 & 0.3760 & 2.5067 & 9 \\
\hline 5.0075 & 11.6811 & 0.0450 & 0.3 & 10 \\
\hline
\end{tabular}


In this paper, the results of first four random positions of the target nodes are shown. Further the position changing process will go on. Table- 3 shows the coordinates of localized nodes with error for first movement. Similar is the case for next movements. Table$4,5,6,7,8,9$ shows the target node coordinates and estimated node coordinates with error for second, third and fourth movement.

Table 4. Target Coordinates for Second Movement

\begin{tabular}{|l|l|l|}
\hline Node No. & Target Coordinates for Second Movement \\
\hline & X & Y \\
\hline 1 & 11.0461 & 5.1304 \\
\hline 2 & 7.4971 & 6.9293 \\
\hline 3 & 0.3412 & 12.7882 \\
\hline 4 & 6.5250 & 11.5317 \\
\hline 5 & 4.0251 & 6.4436 \\
\hline 6 & 5.8440 & 11.9038 \\
\hline 7 & 8.0619 & 2.0740 \\
\hline 8 & 7.6356 & 0.5425 \\
\hline 9 & 8.1157 & 3.6720 \\
\hline 10 & 14.9904 & 5.1436 \\
\hline
\end{tabular}

Table 5. Efficiently Localize Node for Second Movement

\begin{tabular}{|l|l|l|l|l|}
\hline \multicolumn{4}{|l|}{ Efficiently Localize Node for Second Movement } \\
\hline $\begin{array}{l}\text { Localize Node } \\
\text { Coordinates }\end{array}$ & Error & \% Error & $\begin{array}{l}\text { Node } \\
\text { Number }\end{array}$ \\
\hline 11.6081 & 5.3307 & 0.5966 & 3.977333 & 1 \\
\hline 8.0638 & 6.6697 & 0.6234 & 4.156 & 2 \\
\hline 6.5300 & 11.5160 & 0.7880 & 5.253333 & 6 \\
\hline 7.6720 & 1.9827 & 0.4004 & 2.669333 & 7 \\
\hline 7.7354 & -0.3933 & 0.9411 & 6.274 & 8 \\
\hline 7.2806 & 3.6735 & 0.8351 & 5.567333 & 9 \\
\hline
\end{tabular}

Table 6. Target Coordinates for Third Movement

\begin{tabular}{|l|l|l|}
\hline Node No. & \multicolumn{2}{|l|}{ Target Coordinates for Third Movement } \\
\hline & X & Y \\
\hline 1 & 7.6799 & 7.6557 \\
\hline 2 & 14.5925 & 14.9414 \\
\hline 3 & 5.1344 & 0.9149 \\
\hline 4 & 13.0788 & 1.8597 \\
\hline 5 & 1.5352 & 8.4485 \\
\hline 6 & 3.3361 & 14.4627 \\
\hline 7 & 12.4950 & 7.3199 \\
\hline 8 & 13.7347 & 9.2047 \\
\hline 9 & 10.7554 & 4.4644 \\
\hline 10 & 0.4736 & 1.8604 \\
\hline
\end{tabular}


Table 7. Efficiently Localize Node for Third movement

\begin{tabular}{|l|l|l|l|l|}
\hline \multicolumn{4}{|l|}{ Efficiently Localize Node for Third movement } \\
\hline $\begin{array}{l}\text { Localize Node } \\
\text { Coordinates }\end{array}$ & Error & \% Error & $\begin{array}{l}\text { Node } \\
\text { Number }\end{array}$ \\
\hline 14.8125 & 14.7255 & 0.3082 & 2.054667 & 2 \\
\hline 13.3130 & 1.8232 & 0.2370 & 1.58 & 4 \\
\hline 0.5802 & 1.8299 & 0.1109 & 0.739333 & 10 \\
\hline
\end{tabular}

Table 8. Target Coordinates for Fourth Movement

\begin{tabular}{|l|l|l|}
\hline Node No. & \multicolumn{2}{|l|}{$\begin{array}{l}\text { Target Coordinates for Fourth } \\
\text { Movement }\end{array}$} \\
\hline & X & Y \\
\hline 1 & 3.5740 & 11.5733 \\
\hline 2 & 9.6865 & 12.1355 \\
\hline 3 & 3.6317 & 0.2821 \\
\hline 4 & 7.9248 & 14.5462 \\
\hline 5 & 12.3410 & 4.1723 \\
\hline 6 & 7.6039 & 11.9473 \\
\hline 7 & 5.1484 & 9.6106 \\
\hline 8 & 1.0323 & 1.2608 \\
\hline 9 & 9.9666 & 8.7105 \\
\hline 10 & 5.3230 & 0.1392 \\
\hline
\end{tabular}

Table 9. Efficiently Localize Node for Fourth Movement

\begin{tabular}{|l|l|l|l|l|}
\hline \multicolumn{4}{|l|}{ Efficiently Localize Node for fourth movement } \\
\hline $\begin{array}{l}\text { Localize Node } \\
\text { Coordinates }\end{array}$ & Error & \% Error & $\begin{array}{l}\text { Node } \\
\text { Number }\end{array}$ \\
\hline 3.0766 & 11.1297 & 0.6665 & 4.443333 & 1 \\
\hline 7.7905 & 14.6933 & 0.1992 & 1.328 & 4 \\
\hline 12.0334 & 3.7448 & 0.5267 & 3.511333 & 5 \\
\hline 7.1986 & 12.1480 & 0.4523 & 3.015333 & 6 \\
\hline 4.5624 & 10.1850 & 0.8206 & 5.470667 & 7 \\
\hline 0.2198 & 1.5948 & 0.8785 & 5.856667 & 8 \\
\hline
\end{tabular}

Figure-4 Shows the MATLAB simulation results of Anchor node positions and moving target nodes positions at first four counts. The location error, i.e., distance between actual and estimated target nodes is shown for proposed method. To evaluate the performance of the proposed localization algorithm, performance indices like maximum/minimum localization error are considered and results are summarized. For mobility based scenarios, the convergence time of the algorithm should be less, i.e., the convergence should be fast. The algorithm used in this paper has very less convergence time. The average convergence time for PSO at particular position of the anchor and target node is 0.0844 seconds. The average localization error for first ten movements is graphically represented by Figure-5.

The simulation results for static environment having at least three anchor nodes are given in [14]. However, due to consideration of less iterations to reduce computational time (For mobility based scenarios computational time should be very less), the accuracy is bit less but the localization is done using single anchor node only (not by three physical anchors) 
in mobile environment. Also, algorithm used in this paper is better for other challenges like Line of Sight, Localization in mobile scenario and Energy consumption during localization.

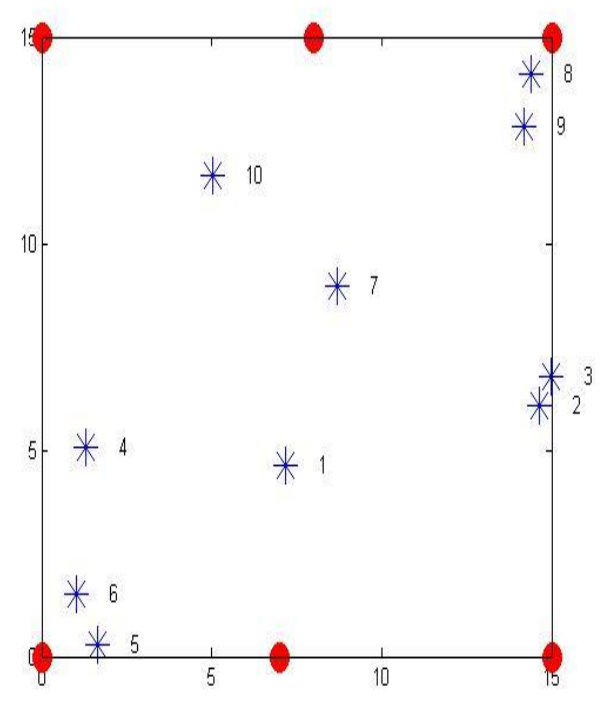

(a)

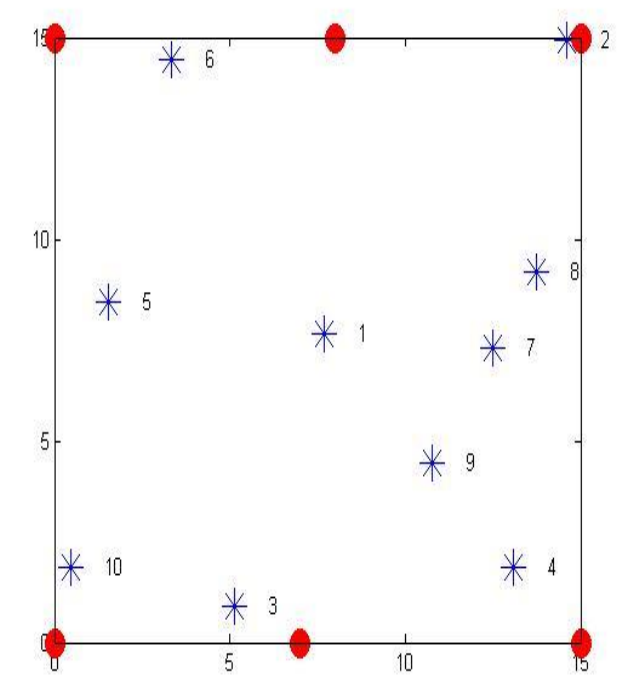

(c)

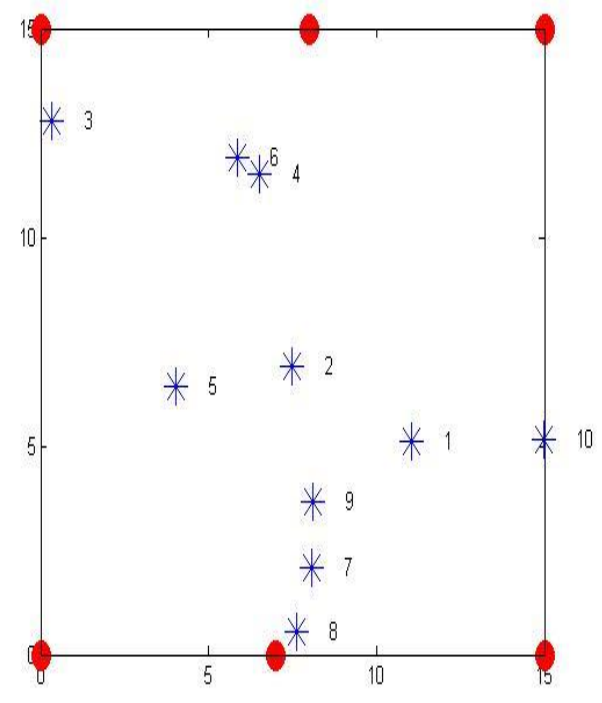

(b)

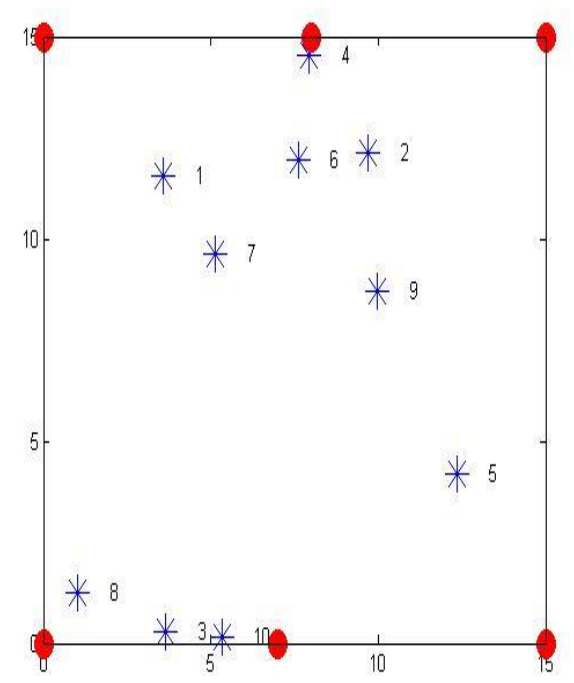

(d)

\begin{tabular}{|cc|}
\hline $\begin{array}{c}\text { Anchor Nodes Deployed at Edges of } \\
\text { Sensing Field }\end{array}$ & $*$ \\
Target Nodes Moving \\
Randomly In the Sensing
\end{tabular}

Figure 4. (a) Positions of Target Nodes for First Movement (b) Positions of Target Nodes for Second Movement (c) Positions of Target Nodes for Third Movement (d) Positions of Target Nodes for Forth Movement 


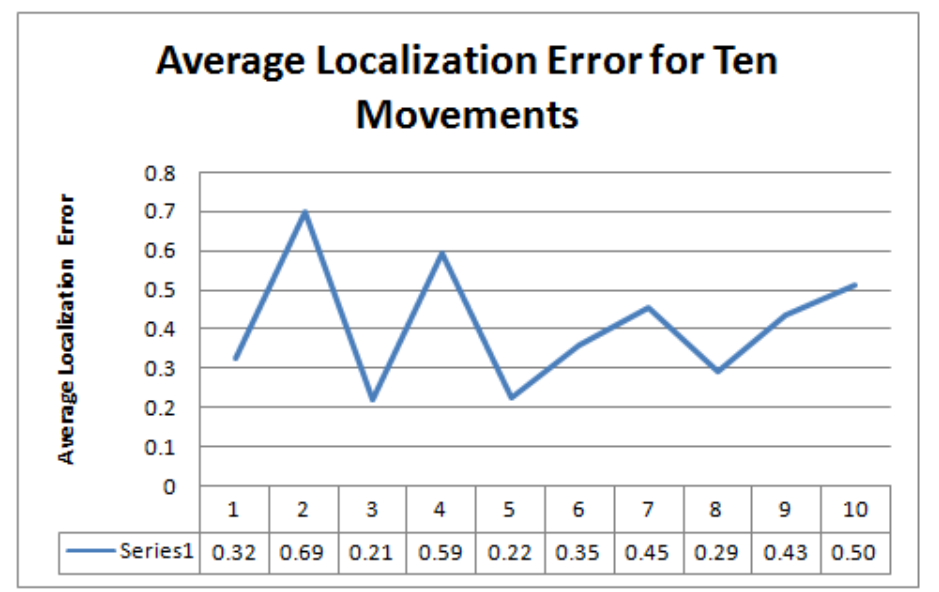

Figure 5. Average Localization Error for Ten Movements

\section{Conclusion and Future Scope}

In this paper, a bio inspired localization algorithm is proposed using application of particle swarm optimization in dynamic wireless sensor networks. The proposed algorithm is divided into two phased. In the first phase, the distance is calculated between anchor and target node, further, virtual anchor nodes are projected inside the sensing field with some angles at a distance equal to anchor and in-range target node. In the second phase, Centroid is calculated and PSO based optimization algorithm is used for location error optimization. The proposed algorithm can be used for the various applications in logistics and military. Further the proposed algorithms may be implemented for 2D/3D centralized localization and range free multi-hop localization for mobile targets or mobile anchors. To achieve more accuracy, a hybrid algorithm can be proposed and different types of trajectories can be used.

\section{References}

[1] F. Akyildiz, W. Su, Y. Sankarasubramaniam and E. Cayirci, "Wireless sensor networks: a survey", Computer networks, vol. 38, no. 4, (2002), pp. 393-422.

[2] H. Karl and A. Willig, "Protocols and architectures for wireless sensor networks", John Wiley and Sons, (2007).

[3] M. Rudafshani and S. Datta. "Localization in wireless sensor networks", 6th International Symposium on Information Processing in Sensor Networks, 2007. IPSN 2007, IEEE, (2007), pp. 51-60.

[4] A. Awad, T. Frunzke and F. Dressler, "Adaptive distance estimation and localization in wsn using rssi measures", 10th Euromicro Conference on Digital System Design Architectures, Methods And Tools, 2007. DSD 2007. IEEE, (2007), pp. 471-478.

[5] G. Di, "A distributed aoa based localization algorithm for wireless sensor networks", Journal of Computers, vol. 3 , no. 4.

[6] P. D. Tinh, T. Noguchi and M. Kawai, "Localization scheme for large scale wireless sensor networks", International Conference on Intelligent Sensors, Sensor Networks and Information Processing, 2008. ISSNIP 2008. IEEE, (2008), pp. 25-30.

[7] K.-Y. Cheng, K.-S. Lui and V. Tam, "Hybloc: Localization in sensor networks with adverse anchor placement", Sensors, vol. 9, no. 1, (2009), pp. 253-280.

[8] G. Mao, B. Fidan and B. D. Anderson, "Wireless sensor network localization techniques", Computer networks, vol. 51, no. 10, (2007), pp. 2529-2553.

[9] Y. Xu, O. Yi, Z. Le, J. Ford and F. Makedon, "Mobile anchor-free localization for wireless sensor networks", Lecture Notes in Computer Science, vol. 4549, (2007), pp. 96.

[10] G. Han, C. Zhang, J. Lloret, L. Shu and J. JPC Rodrigues, "A mobile anchor assisted localization algorithm based on regular hexagon in wireless sensor networks", The Scientific World Journal, vol. 2014, (2014).

[11] V. Garg and M. Jhamb, "Tracking the location of mobile node in wireless sensor network", International Journal of Advanced Research in Computer and Communication Engineering, vol. 2, no. 6.

[12] R. V. Kulkarni and G. K. Venayagamoorthy, "Particle swarm optimization in wireless-sensor networks: A brief survey", IEEE Transactions on Systems, Man, and Cybernetics, Part C (Applications and Reviews), vol. 41, no. 2, (2011), pp. 262-267. 
[13] A. Gopakumar and L. Jacob, "Localization in wireless sensor networks using particle swarm optimization", in: IET International Conference on Wireless, Mobile and Multimedia Networks, IET, (2008), pp. 227230.

[14] A. Kumar, A. Khosla, J. S. Saini and S. Singh, "Meta-heuristic range based node localization algorithm for wireless sensor networks", IEEE International Conference on Localization and GNSS, IEEE, (2012), pp. 1-7.

[15] A. Kumar, A. Khosla, J. S. Saini and S. Singh, "Range-free 3d node localization in anisotropic wireless sensor networks", Applied Soft Computing, vol. 34, (2015), pp. 438-448.

[16] R. J. Kennedy and Eberhart, "Particle swarm optimization", In Proceedings of IEEE International Conference on Neural Networks IV, pages, vol. 1000, (1995).

[17] W. Rehan, S. Fischer, M. Rehan and M. Husain Rehmani, "A Comprehensive Survey on Multichannel Routing in Wireless Sensor Networks", Journal of Network and Computer Applications, (2017).

[18] S. Sivakumar, "Error Minimization in Localization of Wireless Sensor Networks using Fish Swarm Optimization Algorithm”, International Journal of Computer Applications, vol. 159, no. 7, (2017).

[19] V.R. Kulkarni, V. Desai and R. V. Kulkarni, "Multistage localization in wireless sensor networks using artificial bee colony algorithm", In Computational Intelligence (SSCI), 2016 IEEE Symposium Series on., IEEE, (2016), pp. 1-8.

[20] T. LN Nguyen and Y. Shin, "Multiple target localization in wsns based on compressive sensing using deterministic sensing matrices", International Journal of Distributed Sensor Networks, (2015).

[21] G. Han, J. Jiang, C. Zhang, T. Q. Duong, M. Guizani and G. K. Karagiannidis, "A survey on mobile anchor node assisted localization in wireless sensor networks", IEEE Communications Surveys \& Tutorials, vol. 18, no. 3, (2016), pp. 2220-2243.

[22] M. Singh and P. Mohan Khilar, "A Range Free Geometric Technique for Localization of Wireless Sensor Network (WSN) Based on Controlled Communication Range”, Wireless Personal Communications, vol. 94, no. 3, (2017), pp. 1359-1385.

[23] H. Ahmadi, F. Viani, A. Polo and R. Bouallegue, "Learning ensemble strategy for static and dynamic localization in wireless sensor networks", International Journal of Network Management, (2017). 
International Journal of Grid and Distributed Computing Vol. 10, No. 10 (2017) 